\title{
SEMANTIC ANALYSIS APPROACH TO STUDYING DESIGN PROBLEM SOLVING
}

\author{
Georgiev, Georgi V. (1); Georgiev, Danko D. (2) \\ 1: Center for Ubiquitous Computing, University of Oulu, Finland; 2: Institute for Advanced Study, \\ Varna, Bulgaria
}

\begin{abstract}
To objectively and quantitatively study transcribed protocols of design problem solving conversations, we propose a semantic analysis approach based on dynamic semantic networks of nouns constructed with WordNet 3.1 lexical database. We examined the applicability of the semantic approach focused on a dynamic evaluation of the design problem solving process in educational settings. Using a case of realworld design problem-solving conversations, we show that the approach is able to determine the time dynamics of semantic factors such as level of abstraction, polysemy or information content, and quantify convergence/divergence of semantic similarity in design conversations between students, instructors and real clients. The approach can also be used to evaluate the aforementioned semantic factors for successful and unsuccessful ideas generated in the process of design problem solving, or to assess the effect of external feedback on the developed design solution. The proposed semantic analysis approach allows fast computation of the semantic factors in real time thereby demavonstrating a potential for both monitoring and support of the design problem solving process.
\end{abstract}

Keywords: Design cognition, Design process, Human behaviour in design, Semantic analysis, Design conversations

Contact:

Georgiev, Georgi V.

University of Oulu

Center for Ubiquitous Computing

Finland

georgi.georgiev@oulu.fi

Cite this article: Georgiev, G.V., Georgiev, D.D. (2019) 'Semantic Analysis Approach to Studying Design Problem Solving', in Proceedings of the 22nd International Conference on Engineering Design (ICED19), Delft, The Netherlands, 5-8 August 2019. DOI:10.1017/dsi.2019.188 


\section{INTRODUCTION}

Language is a powerful resource for studying design problem solving. Extracting meaningful results about the cognitive processes underlying human creativity from transcribed protocols of design conversations, however, is a challenging task because not all aspects of human creative skills are verbalized or represented at a consciously accessible level (Boden, 2004). Semantic networks overcome the latter issue by providing a structured representation of not only the explicitly verbalized concepts contained in the conversations (Georgiev et al., 2008; Georgiev et al., 2010), but also of the inexplicitly imaged virtual concepts that connect the verbalized concepts inside a standardized lexical database (Yamamoto et al., 2009). In the approach presented here, we use WordNet 3.1 as a lexical database for the construction of semantic networks containing only nouns. Working with a single lexical category (nouns) is motivated by WordNet structure, which is comprised of four subnets (for nouns, verbs, adjectives or adverbs) that are sparse in cross-subnet pointers (Fellbaum 1998). Our choice to work with nouns is further justified by the fact that nouns form the largest and deepest hierarchical taxonomy in WordNet. In addition, previous research has demonstrated that noun phrases are useful surrogates for measuring early phases of the mechanical design process (Mabogunje and Leifer 1997), noun networks stimulate the generation of ideas in creative problem solving (Segers et al., 2005), noun-noun combinations and noun-noun relations are essential ingredients of displayed creative thought in design (Dong 2009), and dissimilarity of noun-noun combinations enhances creative performance through yielding emergent properties of generated ideas (Wilkenfeld and Ward 2001). Noteworthy, noun senses are not disambiguated for the construction of semantic networks because the nouns employed in the description of creative design ideas may acquire new senses different from dictionary-defined ones and polysemy may lead to association of ideas, which were thought to be unrelated previously (Taura and Nagai 2013; Georgiev and Taura 2014). The efficacy of semantic networks of nouns for computational reconstruction of difficult-to-observe processes in design-thinking and exploration of creativity in conceptual design was shown in previous studies using different sets of experimental data (Georgiev et al., 2008; Yamamoto et al., 2009; Georgiev et al., 2010; Taura et al., 2012; Georgiev and Georgiev 2018).

\subsection{Subjective quantitative methods to study design protocols}

Analysis of temporal aspect of protocols of the design process has been beneficial for gaining insights into the generation of ideas and solutions. Investigating design with linkography (Goldschmidt, 2014; Goldschmidt et al., 2014), a method for analysing of decisions and activities that occur in the process of design thinking, requires trained design experts to parse the design conversations into elementary steps called design moves, which may be then reticulated with the use of backlinks to previous moves or forelinks to future moves. The linkography analysis of divergent and convergent thinking in design problem solving conversations highlights their frequent and concurrent occurrence, and their interwoven role for design creativity (Goldschmidt, 2016). Information theoretic development to assessing creativity using linkography has shown that the Shannon entropy of the linkograph may not be correlated with the outcome of design, however, the second time derivative of the entropy curve is positive for high-scoring design sessions and negative for low-scoring ones (Kan and Gero 2017).

\subsection{Objective quantitative methods to study design protocols}

Semantic approaches such as lexical chain analysis have been used to differentiate discontinuities in agreement in design problem solving (Dong, 2007; Dong, 2009). A latent semantic approach has been successful in detailing forms of language for expressing judgments or identifying semantic resources in linguistic appraisals in the context of design conversations (Dong, 2009). Moreover, semantic-based approaches to analysing design (Dong, 2005; Dong, 2007) emphasize that word relations are essential for understanding design thinking. In particular, noun-noun combinations and noun-noun relations play an essential role in design. For example, specific interpretations of a novel noun-noun phrase (such as a new concept that is not included in the two phrases but that inherits specific characteristics of both) and recognition of differences that are unrelated to the common structure characterize the creative concept generation process (Nagai et al., 2009). WordNet has been used to quantify the associative processes occurring in divergent thinking (Acar and Runco 2014). 


\subsection{Semantic analysis with semantic networks}

By providing a structural representation of knowledge, semantic networks represent explicit and inexplicit concepts and context. Recent research developed a number of approaches to analyse design based on semantic networks (Yamamoto et al., 2009; Georgiev et al., 2010; Georgiev and Georgiev 2018). Semantic-based graphs are used to quantify the collective wisdom in a design class (Nickerson et al., 2013). Semantic-based algorithmic methods have been used to score creativity-related divergent thinking (Beketayev and Runco 2016). The thinking process of designers was studied based on dynamics of networks of linked data where the generated dynamic network of edges and nodes represents the information seeking activity-linking sources to activities in time instances, allowing to analyse patterns of design activity, qualitatively and quantitatively, with respect to time (Cash et al., 2014). Simulation of difficult-to-observe design thinking processes and investigation of creativity in conceptual design with the use of semantic networks of nouns was performed in previous experimental studies (Taura et al., 2012). The main advantages of using dynamic semantic networks to analyse transcribed textual data from real conversations are the applicability of the method for studying any cognitive processes occurring in the human mind, including processes that cannot be parsed into design moves, and the robust computation of objective information theoretic measures. Hereafter, we denote the methods that use dynamic semantic networks to analyse transcribed textual data as a 'semantic analysis approach'.

The aim of this study is to explore the applicability of semantic analysis approach for dynamic evaluation of the design problem solving process. We discuss the semantic analysis approach as a method to analyse and gain insights in design problem solving and a tool for objective evaluation of difficult-to-observe cognitive processes.

In the next section, we elaborate on the semantic analysis approach and introduce definitions of the terms. In section 3, we focus on the method of identification of the successful and unsuccessful ideas. In section 4, we describe the evaluation of design process and its dynamics. Sections 5 and 6 introduce the case study demonstrating the approach and the results, followed by discussion and conclusion.

\section{SEMANTIC ANALYSIS APPROACH}

\subsection{Construction of semantic networks of nouns}

For the construction of semantic networks of nouns, textual data of the design problem-solving conversations were processed with the use of part-of-speech tagging performed by the Natural Language Toolkit (NLTK) (Bird et al., 2009) with the TextBlob library (Loria, 2016). Nouns were then extracted and processed with Python scripts to convert the plural forms to singular. Nouns not listed in WordNet 3.1 were excluded. Lastly, we analysed the constructed semantic networks with WordGraph 3.1, a toolset that implements the WordNet 3.1 hypernym-hyponym hierarchy of nouns as a directed acyclic graph in Wolfram Mathematica.

\subsection{Words, meanings and semantic measures in WordNet 3.1}

The hypernym-hyponym hierarchy between noun synsets (sets of one or more synonyms) organizes WordNet 3.1 into a directed acyclic graph of words and meanings (Figure 1). The core of the graph is formed by meaning vertices, which are encoded with numeric code, e.g. M00021445 with synset \{food, nutrient\}, M05819240 with synset \{food, food_for_thought, intellectual_nourishment $\}$ or M07571428 with synset \{food, solid_food\}. To utilize the efficient algorithms inbuilt in Wolfram Mathematica for computing of graph-theoretic measures, word vertices could either subsume meaning vertices or be subsumed by meaning vertices depending on the computed semantic measure.

\subsubsection{Abstraction of words}

Abstraction accounts for how generalized the word node is compared to the most specific instance. The abstraction of a word $x$ in WordNet 3.1 is computed as the complement to unity of the shortest path distance measured in edges from the root meaning vertex to a meaning vertex subsuming the word $x$ divided by the maximal shortest path distance from the root in the core graph of meanings. Because the depth of a word $x$ is the number of vertices on the shortest path from the root, which is $1+$ the number of edges, the abstraction is $\operatorname{Abstraction}(x)=1-\operatorname{Depth}(x)-1 /$ Max_depth -1 (Figure 1A). 

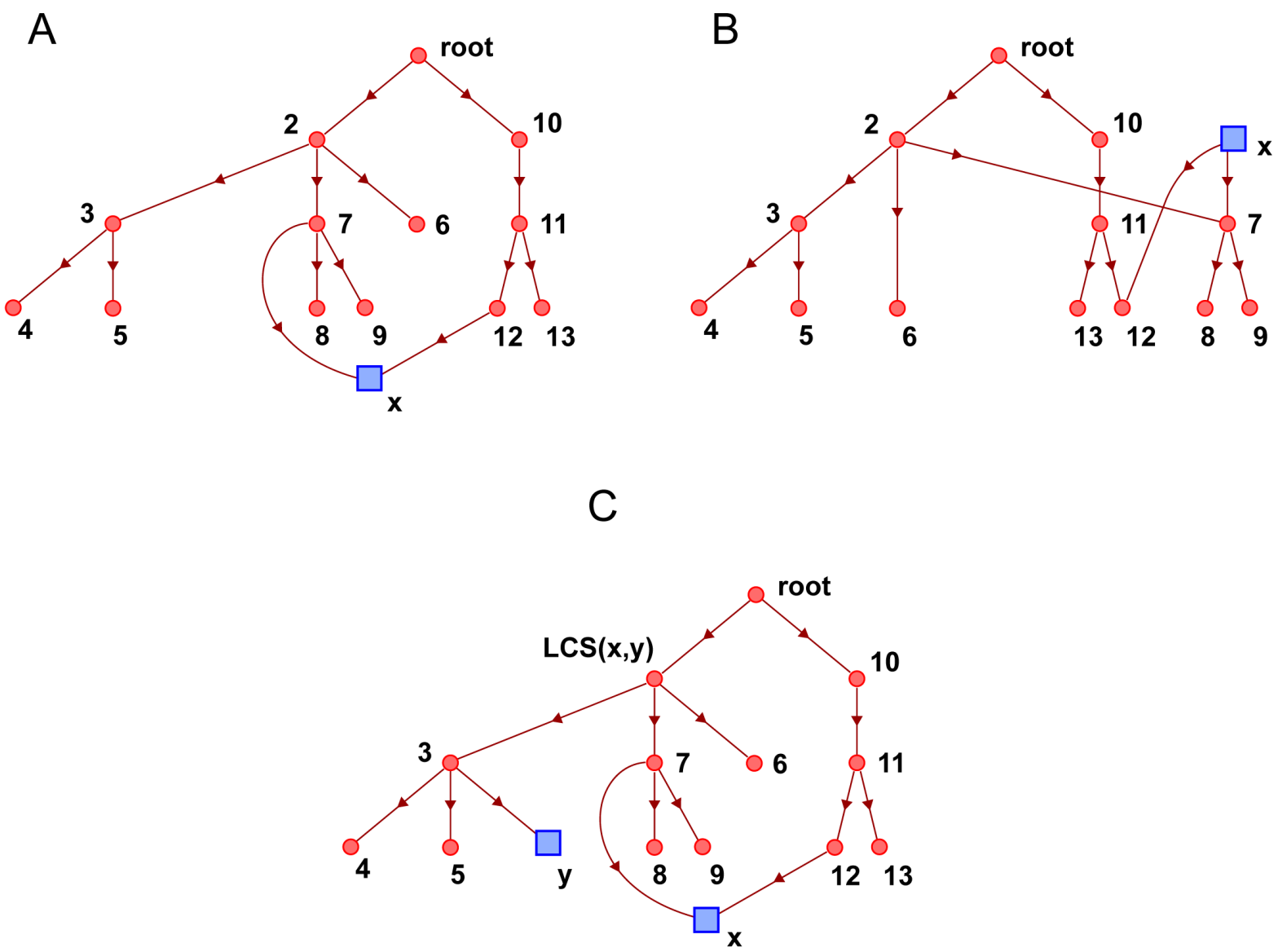

Figure 1. Semantic measures for words in WordNet 3.1 fragment composed of meaning vertices (circles) and word vertices (squares). For different measures the words either subsume meanings or are subsumed by the meanings. (A) Polysemy $(x)=2$; Depth $(x)=3$; Abstraction $(x)=0.67$; $(B)$ |Leaves $(x)$ | = 3; Information Content $(x)=0.44$; (C) LCS $(x, y)$; |Leaves [LCS $(x, y)]$ ] = 5; Semantic similarity $(x, y)=0.17$. Modified from (Georgiev and Georgiev 2018).

Different words in a synset can have different level of abstraction. For example, the abstraction of 'food' is 0.78 , whereas the abstraction of 'food_for_thought' is 0.72 .

\subsubsection{Polysemy of words}

The polysemy counts how many meanings the word has. The polysemy of a word $x$ in WordNet 3.1 is the number of meaning vertices that are directly adjacent to $x$ in the graph (Figure 1B). For example, the polysemy of 'food' is 3, whereas the polysemy of 'food_for_thought' is 1 .

\subsubsection{Information content of words}

The information content is the bits of information carried by a word node inside the graph. The information content of a word in WordNet 3.1 is computed from the number of subsumed leaf meaning vertices and the total number of such leaves in the graph (Figure 1B) (Blanchard et al., 2008). The normalized formula for information content is $\operatorname{IC}(x)=1-\log |\operatorname{Leaves}(x)| / \log \mid \operatorname{Max} \_$leaves $\mid$. For example, IC of 'food' is 0.32 , whereas IC of 'food_for_thought' is 1 .

\subsubsection{Semantic similarity of word pairs}

The semantic similarity quantifies how alike two word nodes are. The semantic similarity of a word pair $(x, y)$ is the information content of the lowest common subsumer of the two words $\operatorname{LCS}(x, y)$ (Figure 1C), which is a meaning vertex in the graph (Resnik, 1999). For example, LCS of 'food' and 'food_for_thought' is M05819240 with synset \{food, food_for_thought, intellectual_nourishment , and the semantic similarity is IC(M05819240) $=1$; LCS of 'food' and 'grass' is M00021445 with synset $\{$ food, nutrient $\}$, and the semantic similarity is $\mathrm{IC}(\mathrm{M} 00021445)=0.36$. 


\section{EVALUATION OF IDEAS: SUCCESSFUL AND UNSUCCESSFUL IDEAS}

Successful and unsuccessful design solutions in relation to the level of expertise and particular modes of reasoning have been identified as critical in the design process (Casakin and Goldschmidt 1999). Moreover, ultimately the successful designs have the potential for innovation (Badke-Schaub, 2004). In this study, we make a distinction of periods in the design conversations that pertain to the particular design idea. Overall, design conversations were divided into two groups: those related to unsuccessful ideas and those related to successful ideas. According to the criterion applied by us, unsuccessful ideas were ideas that had not been developed to the end or had been disregarded in the problem-solving process, whereas successful ideas were those that had been developed to the end (Georgiev and Georgiev 2018). For each design task, only one of the generated ideas was considered to be successful. The same conversation was divided into several parts that pertained to one or more unsuccessful ideas, and a part that pertained to the successful idea. When two ideas were compared in one sentence, the sentence was considered to belong to the idea that was main for the comparison (Georgiev and Georgiev 2018). In Figure 2 is shown the division of the conversations per ideas, which were generated during the design process of one student from the DTRS10 dataset (Adams and Siddiqui 2013; Adams, 2015). There are six unsuccessful ideas, analysed as one part and one successful idea, analysed as the other part. The ideas that are generated during the design problem solving process, in this case, are 'Flower Petal', 'Stacking Peacock', 'Shift (Shifting)', 'Organic', 'Negative Space', 'Wedges', and 'Metal Piece'. Ultimately, the 'Shift (Shifting)' idea is the successful one, whereas all other ideas are unsuccessful.

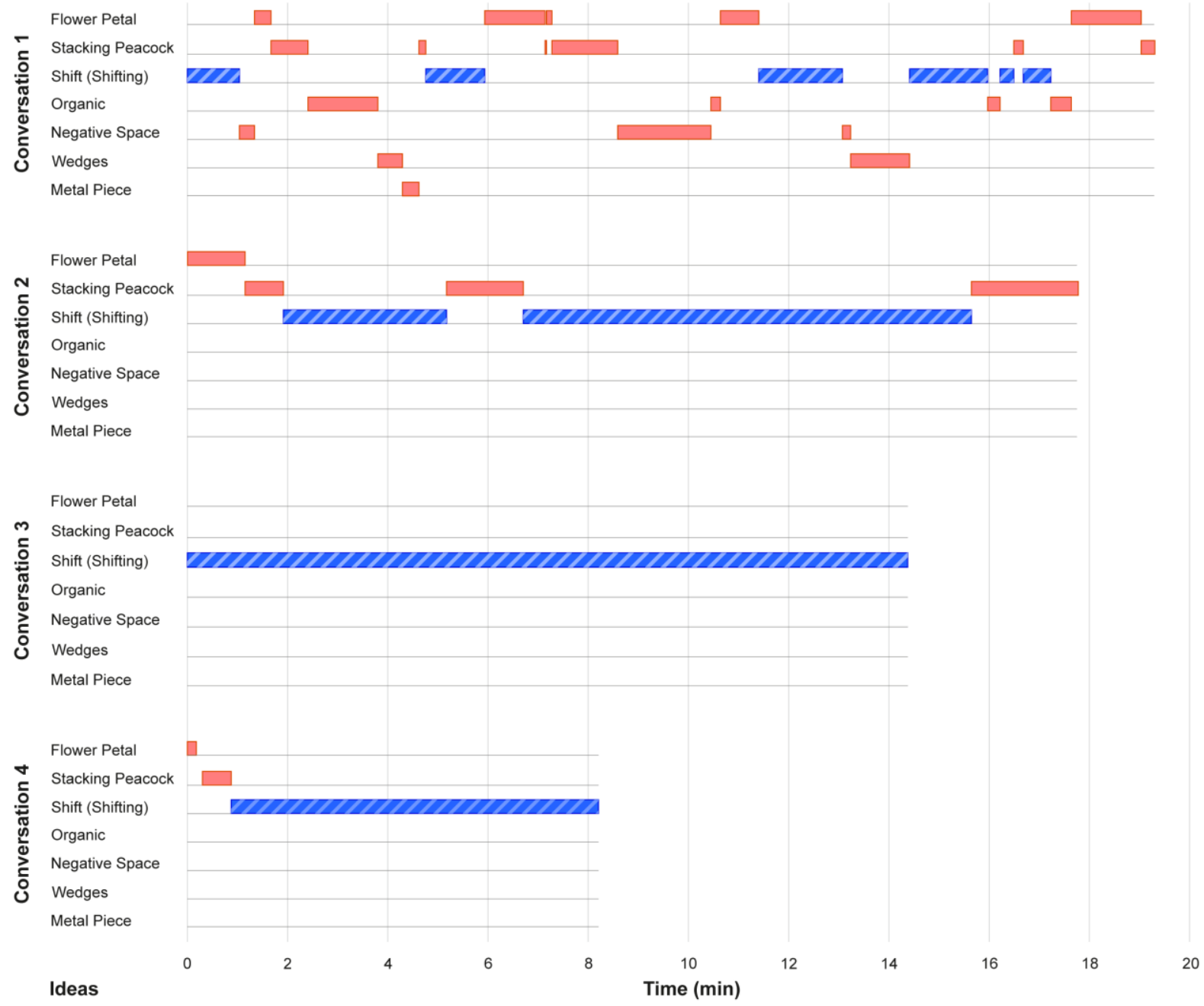

Figure 2. Division of design problem solving conversations into parts pertaining to different design ideas for student J7 of DTRS10 Industrial design junior dataset (the conversations about the successful idea are hatched diagonally). 


\section{EVALUATION OF DESIGN PROBLEM SOLVING PROCESS}

To examine the applicability of the semantic analysis approach for evaluation of different aspects of design problem solving process, we focus on quantification based on participant role, quantification of processes of divergence and convergence as essential in design, as well as on quantification based on wayfinding moments in design conversation, such as receiving outside feedback on design idea.

\subsection{Participant role in design problem solving conversations}

Previously reported comparison between student and instructor speech in the problem-solving conversations did not show statistically significant differences in any of the semantic factors (Georgiev and Georgiev 2018). This result justifies the proposed methodology for analysis of both student and instructor speech jointly with regard to different ideas contained in the conversations.

\subsection{Divergence and convergence in design problem solving conversations}

Creative ideas need to provide both novel (original) and useful (valuable) solutions to the posed design tasks. The success of generated ideas in creative problem solving is judged by the client who selects one of the generated ideas as the most creative one. The prior decisions made by the designer (in this case design student) not to drop the idea in the face of constraints on available resources, however, are also important for the final success of ideas. Thus, success and creativity are intertwined - they are not identical, yet professional designers aim at finding solutions that are both creative and successful.

Previous computational study demonstrated that a program which employs consecutive rounds of divergence and convergence, generates results on a common creativity test that are comparable to the results obtained with humans (Olteţeanu and Falomir 2015). To determine whether divergent or convergent thinking is responsible for the success or failure of ideas, we compared the time dynamics of semantic factors in conversations pertaining to successful or unsuccessful ideas.

\subsection{Dynamics of the design problem solving conversations}

To quantify the dynamics of the design problem solving conversations, we assessed the change of the four semantic factors in time. To obtain three time points for analysis of time dynamics, we joined the conversation transcripts pertaining to each group or idea and then divided the resulting conjoined conversations into three equal parts based on word count (Georgiev and Georgiev 2018). This division was made into whole sentences in such a way that no time point of the conversation contained less than five nouns. Because only nouns in the conversations were used for the construction of semantic networks, each time point had to contain at least five nouns to obtain a proper average semantic similarity (Georgiev and Georgiev 2018). Regarding semantic similarity, convergence in the semantic networks was defined as an increase of the average semantic similarity in time, whereas divergence as a decrease of the average semantic similarity in time.

\subsection{First feedback in design problem solving conversations}

Design conversations were divided into two groups: containing ideas before and after first feedback. The division of conversations was performed at the point of first feedback from the other participant in the conversation, in this case client (a stakeholder that was not a student or appointed as an instructor). To test whether the first feedback from the client influences design problem solving, we compared the dynamics of semantic measures in conversations containing ideas before and after first feedback.

\section{CASE STUDY}

We analysed one of the transcripts of design review conversations recorded in real-world educational settings at Purdue University, West Lafayette, Indiana, in 2013 (Adams and Siddiqui 2013; Adams, 2015). The conversations between design students, instructors, and real clients, with regard to a given design task, consisted of up to 6 sessions in each of 4 conversations in total (Table 1) that included the generation of ideas by the student, external feedback from the client, first evaluation by the client or instructor, and evaluation of the ideas by the client. The example of problem-solving conversations was analysed in terms of successfulness of ideas or first feedback from client using the average values of semantic measures quantifying the level of abstraction (Georgiev and Georgiev 2018), polysemy (Georgiev and Taura 2014; Georgiev and Georgiev 2018) or information content (Blanchard et al., 2008) of each noun, or the semantic similarity (Resnik, 1999) between any two nouns in the constructed semantic networks based on WordNet 3.1 (Fellbaum, 1998). To examine the semantic 
analysis approach, we took the data of one student ( $\mathrm{J} 7$, the last in the particular subset) of the Industrial design junior DTRS10 dataset (Georgiev and Georgiev 2018). The task for the junior students called for the design of "Impromptu" seating places for a real client. The aim was to provide solutions to collaborative work environments, and be versatile in corporate and vertical market segments (For an example of a design outcome in terms of ideas, see Figure 2).

Table 1. Discussion sessions of ideas of student J7 of DTRS10 Industrial design junior dataset.

\begin{tabular}{|c|c|c|c|c|}
\hline & Conversation 1 & Conversation 2 & Conversation 3 & Conversation 4 \\
\hline Flower Petal / Unsuccessful & 5 sessions & 1 session & - & 1 session \\
\hline Stacking Peacock / Unsuccessful & 6 sessions & 3 sessions & - & 1 session \\
\hline Shift (Shifting) / Successful & 6 sessions & 2 sessions & 1 session & 1 session \\
\hline Organic / Unsuccessful & 4 sessions & - & - & - \\
\hline Negative Space / Unsuccessful & 3 sessions & - & - & - \\
\hline Wedges / Unsuccessful & 2 sessions & - & - & - \\
\hline Metal Piece / Unsuccessful & 1 session & - & - & - \\
\hline
\end{tabular}

\section{RESULTS}

Successful and unsuccessful ideas in the design conversations exhibited distinct time dynamics of semantic factors. The level of Abstraction was lower for successful ideas compared to unsuccessful ideas (Figure 3A). Polysemy also decreased in time for successful ideas (Figure 3B), which was accompanied by increased Information Content (Figure 3C). The divergence of Semantic Similarity for successful ideas was contrasted by the manifested convergence for unsuccessful ideas, implicating a role of divergent (convergent) thinking in the success (failure) of generated design ideas (Figure 3D). The parts of design conversations before and after first feedback from the client also exhibited distinct time dynamics but only for 2 of the semantic factors. The level of Abstraction (Figure 4A) and Information Content (Figure 4C) appeared to be almost unchanged, whereas Polysemy (Figure 4B) and Semantic Similarity (Figure 4D) appeared to be increased after first feedback.

A

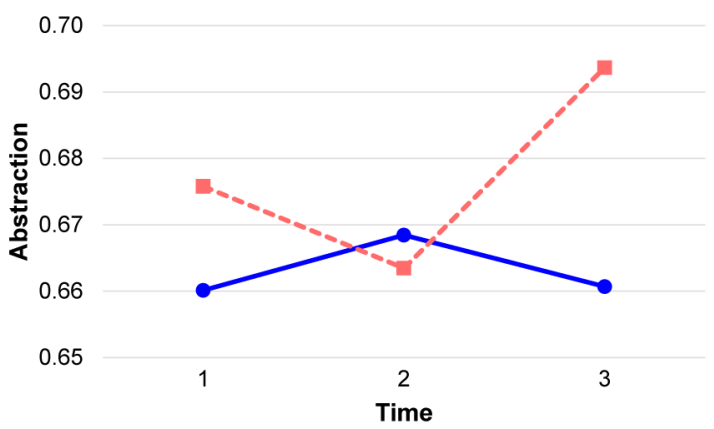

C

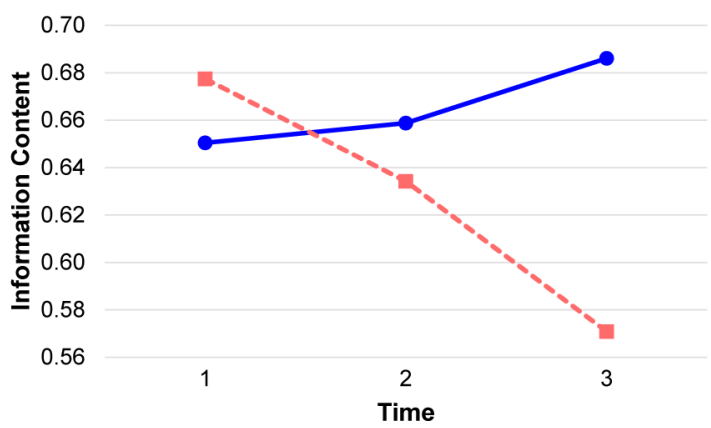

B

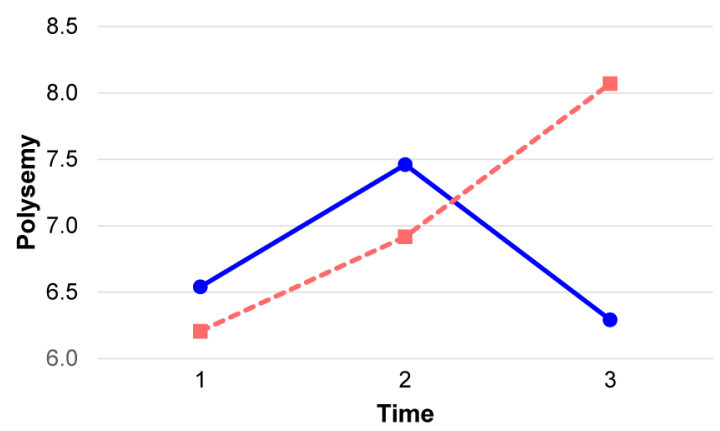

D

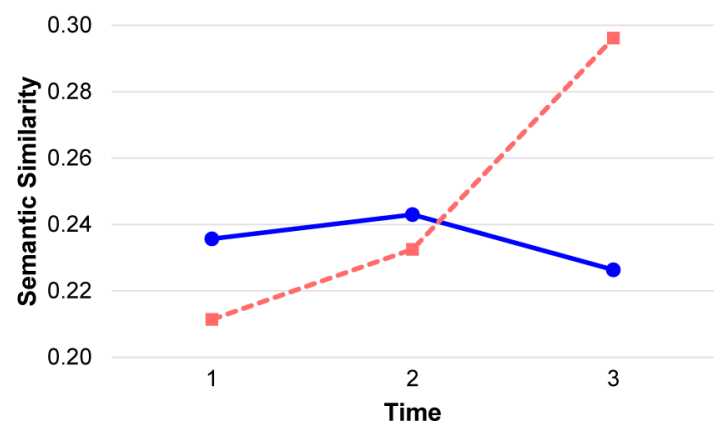

Figure 3. Dynamics of Abstraction (A), Polysemy (B), Information Content (C) and Semantic Similarity (D) for successful (circles, solid line) vs. unsuccessful (squares, dashed line) ideas in the design problem solving conversations of student J7 in the DTRS10 dataset. 
A

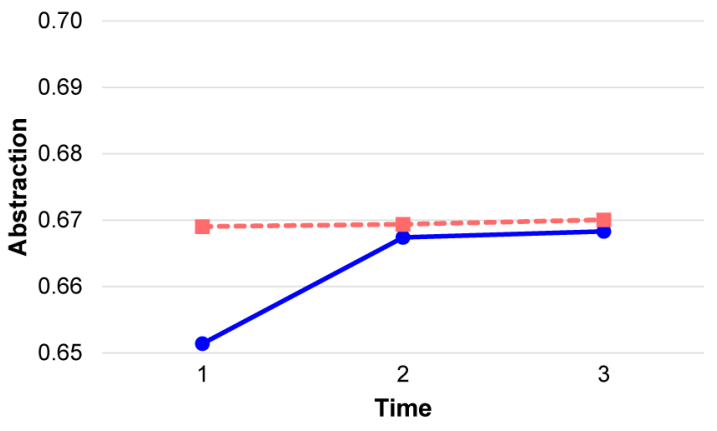

C

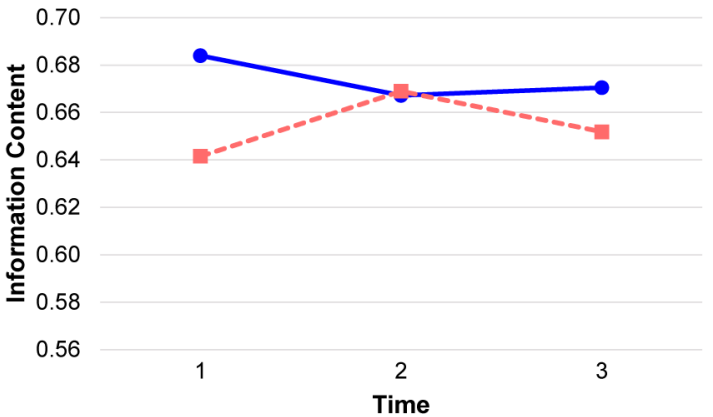

B

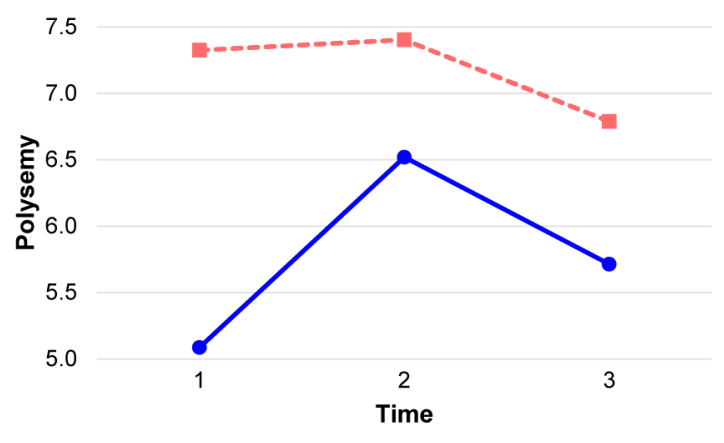

D

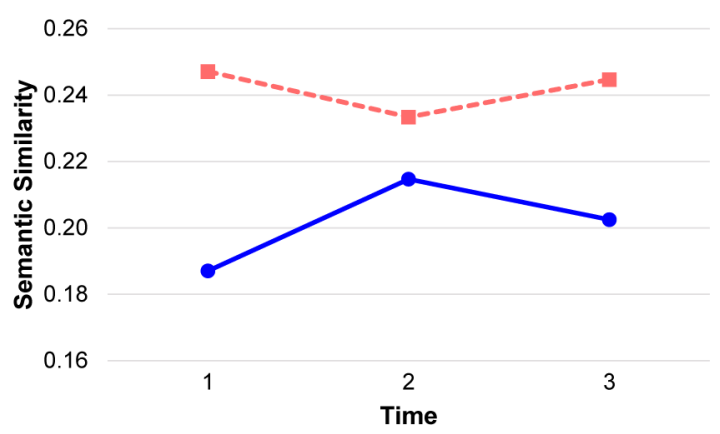

Figure 4. Dynamics of Abstraction (A), Polysemy (B), Information Content (C) and Semantic Similarity (D) before first feedback (circles, solid line) vs. after first feedback (squares, dashed line) from client in the design problem solving conversations of student J7 in the DTRS10 dataset.

\section{DISCUSSION}

\subsection{Quantification of processes in design problem solving}

The presented method for semantic analysis of conversation transcripts based on WordNet 3.1 advances design research by providing a tool for objective evaluation of time dynamics of semantic factors such as level of abstraction, polysemy and information content, and convergence/divergence of semantic similarity in problem-solving conversations in educational settings. The method could be used to quantify the successful and unsuccessful ideas that are generated and developed in the process of problem solving in view of improving student training, creative thinking and skill acquisition. Dynamics of the design problem solving was studied by splitting the conversations into three time points and plotting the data with piecewise linear interpolation. This provides formal quantification of what occurs in the design problem solving process, potentially allowing objective comparisons of design processes. Such comparisons can be made in design education settings, allowing automatic evaluations or feedback. The presented objective quantitative measures based on WordNet 3.1 provide a robust tool for quantitative analysis of verbal data. The semantic analysis approach is sufficiently objective to be used by non-experts, with this addressing one of the shortcomings of the linkography (Hatcher et al., 2018). The convergence or divergence of semantic similarity in verbalized thoughts appears to reflect faithfully the underlying cognitive processes, including convergent (analytical) or divergent (associative) thinking.

\subsection{Application beyond the presented case study}

From a technical point of view, the semantic analysis approach can be implemented in conversational data of similar size. The size constraints are conversations longer than approximately 100 words, with the upper limit set only by computational complexity of all unique semantic similarity pairs of words for the particular part of the conversation analysed. From the point of view of our objectives to explore the applicability of the semantic analysis approach, quantifiable semantic factors such as abstraction, polysemy, information content, and semantic similarity are important for the generation of creative design ideas and for the final outcome of a design task (Wilkenfeld and Ward 2001; Taura and Nagai 2013). It is plausible that there are other semantic factors that are quantifiable with similar approach and show potential for a better understanding of the design process. The limitations of the approach relate to the limitation of lingual communications, such as use of visual images instead of language. 


\section{CONCLUSION}

We examined the applicability of semantic analysis approach to the dynamic evaluation of the design problem solving process, quantification of convergence and divergence of semantic similarity, as well as assessment of time dynamics of level of abstraction, polysemy, and information content. The semantic analysis approach allows for objective evaluation of design problem-solving conversations in educational settings. The approach can be used for detailed examination of successful and unsuccessful ideas, with particular importance for design education. Moreover, the approach allows fast computation of the semantic factors in real time, thus demonstrating the potential for both the analysis and support of the design problem solving process.

\section{ACKNOWLEDGMENTS}

The authors would like to thank Dr. Robin Adams (Purdue University) for the provided access to the DTRS10 dataset under the signed data-use agreement. This research has been partially financially supported by Academy of Finland 6Genesis Flagship (grant 318927).

\section{REFERENCES}

Acar, S. and Runco, M.A. (2014), “Assessing associative distance among ideas elicited by tests of divergent thinking”, Creativity Research Journal, Vol. 26 No. 2, pp. 229-238. http://doi.org/10.1080/10400419.2014.901095

Adams, R.S. (2015). "Design review conversations: The dataset" in Adams, R. S. and Siddiqui, J. A., eds., Analyzing Design Review Conversations, Indiana: Purdue University Press, West Lafayette.

Adams, R.S. and Siddiqui, J.A. (2013), Purdue DTRS - Design Review Conversations Database, XRoads Technical Report, TR-01-13, Indiana: Purdue University, West Lafayette.

Badke-Schaub, P. (2004), "Strategies of experts in engineering design: between innovation and routine behaviour", Journal of Design Research, Vol. 4 No. 2, pp. 125-143. http://doi.org/10.1504/jdr.2004.009837

Beketayev, K. and Runco, M.A. (2016), "Scoring divergent thinking tests by computer with a semantics-based algorithm”, Europe's Journal of Psychology, Vol. 12 No. 2, pp. 210-220. http://doi.org/10.5964/ejop.v12i2.1127

Bird, S., Klein, E. and Loper, E. (2009), Natural Language Processing with Python, Sebastopol, O’Reilly Media, California.

Blanchard, E., Harzallah, M. and Kuntz, P. (2008), “A generic framework for comparing semantic similarities on a subsumption hierarchy" in Ghallab, M., Spyropoulos, C. D., Fakotakis, N. and Avouris, N., eds., ECAI 2008: 18th European Conference on Artificial Intelligence including Prestigious Applications of Intelligent Systems (PAIS 2008), Greece: IOS Press, Patras, pp. 20-24.

Boden, M.A. (2004), The Creative Mind: Myths and Mechanisms, 2nd ed., Routledge, London.

Casakin, H. and Goldschmidt, G. (1999), "Expertise and the use of visual analogy: implications for design education", Design Studies, Vol. 20 N o. 2, pp. 153-175. http://doi.org/10.1016/S0142-694X(98)00032-5

Cash, P., Stanković, T. and Štorga, M. (2014), "Using visual information analysis to explore complex patterns in the activity of designers", Design Studies, Vol. 35 No. 1, pp. 1-28. http://doi.org/10.1016/j.destud.2013.06.001

Dong, A. (2005), "The latent semantic approach to studying design team communication", Design Studies, Vol. 26 No. 5, pp. 445-461. http://doi.org/10.1016/j.destud.2004.10.003

Dong, A. (2007), “The enactment of design through language”, Design Studies, Vol. 28 No. 1, pp. 5-21. http://doi.org/10.1016/j.destud.2006.07.001

Dong, A. (2009), The Language of Design: Theory and Computation, Springer, London.

Fellbaum, C. (1998), WordNet: An Electronic Lexical Database, The MIT Press, Cambridge, Massachusetts.

Georgiev, G.V. and Georgiev, D.D. (2018), "Enhancing user creativity: semantic measures for idea generation", Knowledge-Based Systems, Vol. 151, pp. 1-15. http://doi.org/10.1016/j.knosys.2018.03.016

Georgiev, G.V., Nagai, Y. and Taura, T. (2008), "Method of design evaluation focused on relations of meanings for a successful design”, in Marjanovic, D., Storga, M., Pavkovic, N. and Bojcetic, N., eds., 10th International Design Conference, DESIGN 2008, Dubrovnik, Croatia, May 19-22, 2008, The Design Society, pp. 1149-1158.

Georgiev, G.V., Nagai, Y. and Taura, T. (2010), "A method for the evaluation of meaning structures and its application in conceptual design", Journal of Design Research, Vol. 8 No. 3, pp. 214-234. http://doi.org/10.1504/jdr.2010.032607

Georgiev, G.V. and Taura, T. (2014), "Polysemy in design review conversations" in 10th Design Thinking Research Symposium, Purdue University, West Lafayette, Indiana: Purdue University. 
Goldschmidt, G. (2014), Linkography: Unfolding the Design Process, MIT Press, Cambridge, Massachusetts.

Goldschmidt, G. (2016), "Linkographic evidence for concurrent divergent and convergent thinking in creative design”, Creativity Research Journal, Vol. 28 No. 2, pp. 115-122. http://doi.org/10.1080/10400419.2016.1162497

Goldschmidt, G., Casakin, H., Avidan, Y. and Ronen, O. (2014), “Three studio critiquing cultures: Fun follows function or function follows fun?" in 10th Design Thinking Research Symposium, Purdue University, Indiana: Purdue University, West Lafayette.

Hatcher, G., Ion, W., Maclachlan, R., Marlow, M., Simpson, B., Wilson, N. and Wodehouse, A. (2018), "Using linkography to compare creative methods for group ideation”, Design Studies, Vol. 58, pp. 127-152. http://doi.org/10.1016/j.destud.2018.05.002

Kan, J.W.T. and Gero, J.S. (2017), Quantitative Methods for Studying Design Protocols, Springer, Dordrecht.

Loria, S. (2016), TextBlob: Simplified Text Processing, Center for Open Science, Charlottesville, Virginia.

Mabogunje, A. and Leifer, L.J. (1997), "Noun phrases as surrogates for measuring early phases of the mechanical design process", in 1997 ASME Design Engineering Technical Conferences: DETC '97, Sacramento, California, September 14-17, 1997, American Society of Mechanical Engineers.

Nagai, Y., Taura, T. and Mukai, F. (2009), "Concept blending and dissimilarity: factors for creative concept generation process”, Design Studies, Vol. 30 No. 6, pp. 648-675. http://doi.org/10.1016/j.destud.2009.05.004

Nickerson, J.V., Corter, J.E., Tversky, B., Rho, Y.-J., Zahner, D. and Yu, L. (2013), “Cognitive tools shape thought: diagrams in design”, Cognitive Processing, Vol. 14 No. 3, pp. 255-272. http://doi.org/10.1007/s10339-013-0547-3

Olteţeanu, A.-M. and Falomir, Z. (2015), “comRAT-C: A computational compound Remote Associates Test solver based on language data and its comparison to human performance", Pattern Recognition Letters, Vol. 67 No.1, pp. 81-90. http://doi.org/10.1016/j.patrec.2015.05.015

Resnik, P. (1999), "Semantic similarity in a taxonomy: an information-based measure and its application to problems of ambiguity in natural language", Journal of Artificial Intelligence Research, Vol. 11, pp. 95-130.

Segers, N.M., de Vries, B. and Achten, H.H. (2005), “Do word graphs stimulate design?”, Design Studies, Vol. 26 No. 6, pp. 625-647. http://doi.org/10.1016/j.destud.2005.05.002

Taura, T. and Nagai, Y. (2013), Concept Generation for Design Creativity: A Systematized Theory and Methodology, Springer, London.

Taura, T., Yamamoto, E., Fasiha, M.Y.N., Goka, M., Mukai, F., Nagai, Y. and Nakashima, H. (2012), "Constructive simulation of creative concept generation process in design: a research method for difficultto-observe design-thinking processes”, Journal of Engineering Design, Vol. 23 No. 4, pp. 297-321. http://doi.org/10.1080/09544828.2011.637191

Wilkenfeld, M.J. and Ward, T.B. (2001), "Similarity and emergence in conceptual combination", Journal of Memory and Language, Vol. 45 No. 1, pp. 21-38. http://doi.org/10.1006/jmla.2000.2772

Yamamoto, E., Goka, M., Yusof, N.F.M., Taura, T. and Nagai, Y. (2009), "Virtual modeling of concept generation process for understanding and enhancing the nature of design creativity", in Norell Bergendahl, M., Grimheden, M., Leifer, L., Skogstad, P. and Lindemann, U., eds., 17th International Conference on Engineering Design, Vol. 2, Design Theory and Research Methodology, Palo Alto, CA, August 24-27, 2009, The Design Society, pp. 101-112. 